\title{
FBMC vs OFDM Waveform Contenders for 5G Wireless Communication System
}

\author{
Parnika Kansal, Ashok Kumar Shankhwar\# \\ Department of Electronics, School of Engineering, Harcourt Butler Technical University (HBTU), Kanpur, India \\ Email: parnikakansa193@gmail.com, akshankwar@hbtu.ac.in
}

How to cite this paper: Kansal, P. and Shankhwar, A.K. (2017) FBMC vs OFDM Waveform Contenders for $5 \mathrm{G}$ Wireless Communication System. Wireless Engineering and Technology, 8, 59-70.

https://doi.org/10.4236/wet.2017.84005

Received: August 11, 2017

Accepted: October 18, 2017

Published: October 23, 2017

Copyright @ 2017 by authors and Scientific Research Publishing Inc. This work is licensed under the Creative Commons Attribution International License (CC BY 4.0).

http://creativecommons.org/licenses/by/4.0/

(c) (i) Open Access

\begin{abstract}
The multicarrier transmission techniques have been the most beguiling one for the development of wireless communication systems like $4 \mathrm{G}$, Long Term evolution and now the successor 5G. For 5G or fifth generation wireless communication, Orthogonal Frequency Division Multiplexing (OFDM) and Filter Bank Multi carrier modulation (FBMC) are the dominant waveform contenders. In this research paper, the disadvantages of OFDM have been addressed and it has shown that filter bank multicarrier (FBMC) could be a more effective solution. The comparative analysis of FBMC and OFDM has been performed based on Power Spectral Densities, sub channels, computational complexity and prototype filter comparison simulated using MATLAB.
\end{abstract}

\section{Keywords}

FBMC, OFDM, Multicarrier, Prototype Filter, 5G, LTE

\section{Introduction}

The evolution of internet and the growing demand of high data rate users have given Dynamic spectrum access networks a widespread attention in the recent years. In the DSA networks, multiple nodes contend to employ a shared frequency spectrum. The OFDM technique with cyclic prefix (CP-OFDM) that uses an orthogonal set of subcarriers is by far the most widespread case of multicarrier systems which have been anticipated for the purpose of sharing the various subsets of these subcarriers amid nodes [1] [2] installed for DSA. But, the tight timing and synchronization requirement in OFDM is problematic to attain in practice when the nodes belong to various administrative units. Hence, mutual interference amid the signals of various users is resulted in case of an absence of synchronization. Furthermore, the $\mathrm{CP}$ employed is chastely redundant in terms

\#Ashok Kumar Shankhwar, Associate Professor, HBTU Kanpur. 
of information and significantly diminishes the bandwidth efficiency. The disadvantages of OFDM technique were overcome by a multicarrier communication system called as FBMC first proposed by Saltzberg [3] that delivers an improved spectral shaping of subcarriers than OFDM systems. This is then achieved by the careful designing of the prototype filter, which abridges equalization in the lack of $\mathrm{CP}$ and also promises an additional effectual spectral utilization by diminishing interference across subcarriers [4]. By engaging Offset Quadrature Amplitude Modulation (OQAM), the full capacity of the transmission bandwidth can be attained in FBMC systems. Hence, the main aim of this research paper is to compare the multicarrier techniques OFDM and FBMC techniques for 5G wireless systems. By using MATLAB all the simulations have been performed for comparing the two multicarrier techniques in terms of the Power Spectral Densities, computational complexity and prototype filter comparisons of FBMC and OFDM [5]. This research paper is organized into the following sections-Section 1 gives the introduction of the research paper and highlights the idea behind the comparison of FBMC and OFDM techniques, Section 2 gives the main differences between the two multicarrier techniques with the help of their respective block diagrams, Section 3 discusses about the transmultiplexer configuration of FBMC technique giving the details about the input and outputs, the filter banks used in transmitter and receiver side and the offset quadrature amplitude modulation pre/post processing, Section 4 is about the simulations performed on MATLAB for comparing the FBMC and OFDM techniques in terms of Power spectral densities, computational complexities of the techniques in terms of magnitude response and the magnitude responses of the prototype filters for both techniques, finally Section 5 gives the conclusion of the research paper. Section 5 is followed by Acknowledgements, nomenclature used in the paper and the references.

\section{Differences between OFDM and FBMC Multi Carrier Techniques}

FBMC is an evolved version of OFDM. The modulators of the OFDM technique and FBMC technique are illustrated in Figure 1 [5]. The main difference is the replacement of the OFDM with a multicarrier system based on filter banks, where the IFFT plus $\mathrm{CP}_{\text {in }}$ is substituted by the synthesis filter bank (SFB) whereas

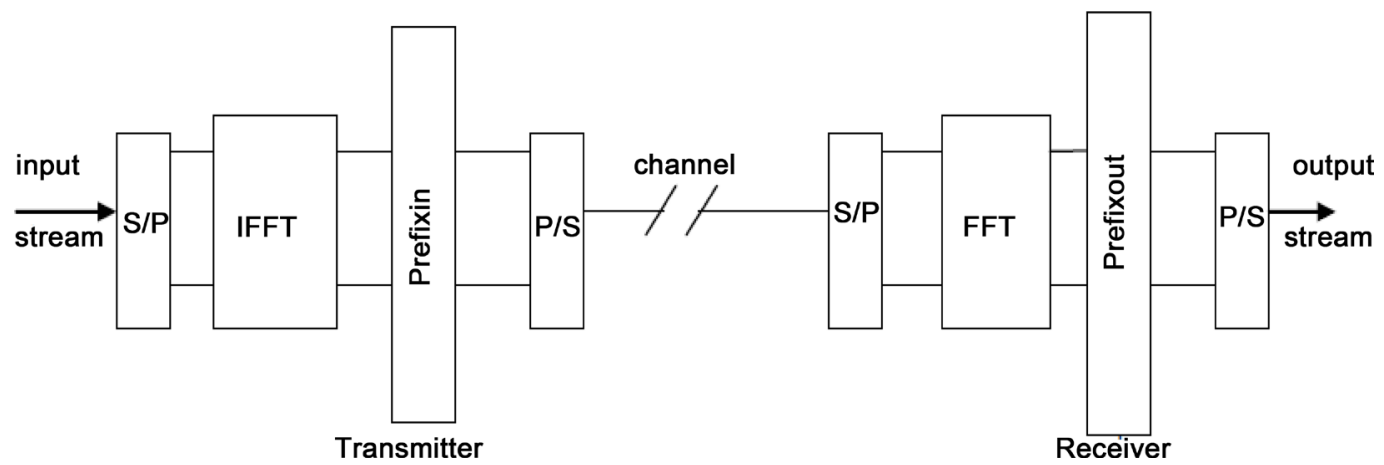

Figure 1. Block diagram of orthogonal frequency division multiplexing [5] [6]. 
Table 1. Major differences between OFDM and FBMC [5].

\begin{tabular}{|c|c|c|}
\hline Property & OFDM & FBMC \\
\hline $\begin{array}{l}\text { Cyclic Prefix } \\
\text { Extension }\end{array}$ & $\begin{array}{l}\text { Cyclic prefix is required in OFDM and } \\
\text { this sacrifices the Bandwidth }\end{array}$ & $\begin{array}{l}\text { Cyclic prefix not required and } \\
\text { this conserves the bandwidth }\end{array}$ \\
\hline Sidelobes & Large and interfering sidelobes & Low sidelobes \\
\hline Synchronisation & $\begin{array}{l}\text { For correct detection, multiple access } \\
\text { interference (MAI) cancellation } \\
\text { should be performed at the receiver }\end{array}$ & $\begin{array}{l}\text { MAI is repressed due to } \\
\text { excellent frequency localisation } \\
\text { of the subcarriers }\end{array}$ \\
\hline Doppler Effect & $\begin{array}{l}\text { Highly sensitive to carrier frequency } \\
\text { offset }\end{array}$ & $\begin{array}{l}\text { Less sensitive and hence } \\
\text { performs significantly with the } \\
\text { increase of the user mobility }\end{array}$ \\
\hline MIMO Systems & $\begin{array}{l}\text { High flexibility while adopting MIMO } \\
\text { techniques }\end{array}$ & Limited flexibility \\
\hline Spectrum Sensing & $\begin{array}{c}\text { Degraded spectrum sensing } \\
\text { performance due to spectral leakage } \\
\text { problem in OFDM }\end{array}$ & $\begin{array}{l}\text { High spectrum sensing } \\
\text { resolution }\end{array}$ \\
\hline $\begin{array}{l}\text { Computational } \\
\text { Complexity }\end{array}$ & It has very low complexity & It has high complexity \\
\hline
\end{tabular}

FFT plus $\mathrm{CP}_{\text {out }}$ is substituted by the analysis filter bank (AFB).The main differences between OFDM and FBMC techniques have been summarized in Table 1 on the properties-cyclic prefix, sidelobes, synchronization, Doppler effect, MIMO systems, Spectrum sensing and computational complexity. The basic principles of OFDM and FBMC are explicated below.

\subsection{Orthogonal Frequency Division Multiplexing (OFDM)}

In OFDM multicarrier system, the frequency spectrum of the subcarriers is overlapped with the least frequency spacing and the orthogonality is attained amid the various subcarriers. In Figure 1, the input stream is subdivided into parallel data streams by means of the serial to parallel $(\mathrm{S} / \mathrm{P})$ converter, that are then passed into an inverse fast Fourier transformation (IFFT) block to produce time sequence of the streams. Consequently, by totalling a cyclic prefix (CP), the OFDM symbol time sequences are extended. The CP is a copy of the latter portion of the symbol that is added in the start of the sequence and should be greater than the network deferral spread in order to diminish the inter symbol interference (ISI) produced by the influx of various OFDM symbols with distinct delay. The resultant digital signal is transformed into analog form and transmitted over the channel [5].

At the receiver end, the signal is reconstructed into digital form and the far Fourier transform (FFT) is achieved in the received streams after eradicating the $\mathrm{CP}$ [5]. Finally, the parallel streams are collected into a single stream as the original transmitted one. Some of the disadvantages of OFDM are enumerated below:

1) Decreased spectral efficiency owing to the $\mathrm{CP}$ employed;

2) High spectral leakage owing to the rectangular windowing; 
3) Interference amid the unsynchronized signal in the neighbouring bands.

\subsection{Filter Bank Multi Carrier}

The FBMC technique overcomes the limitations of OFDM by adding generalized pulse shaping filters which delivers a well localized subchannel in both time and frequency domain. Consequently, FBMC systems have more spectral containment signals and offer more effective use of the radio resources where no $\mathrm{CP}$ is required. In Figure 2 it can be seen that the filterbanks on the transmitter side and the receiver side consist of an array of $\mathrm{N}$ filters that processes $\mathrm{N}$ input signals to give $\mathrm{N}$ outputs. If the inputs of these $\mathrm{N}$ filters are associated together, the system in analogous manner can be measured as an analyzer to the input signal based on each filter characteristics. In Figure 2 the filter bank used at the transmitter side is called synthesis filter bank and the filter bank used in receiver side is called analysis filter bank. As depicted in Figure 2 the input signal is first converted from serial to parallel form and then passed through synthesis filter bank and then it is converted back to serial form after coming out of synthesis bank. After this it can be seen in Figure 2 that in the receiver side after the signal passes through the channel it is converted to parallel form by serial to parallel converter and passed through analysis filter bank. Finally when the output signal is obtained it is again converted to serial form by parallel to serial converter. [7]. Hence the synthesis-analysis configuration depicted in Figure 2 is called transmulitplexer or TMUX and is applied in the MC communication systems [8].

\section{Transmultiplexer Configuration of Filter Bank Multi Carrier}

The core of the FBMC system is the TMUX configuration presented in Figure 3 the main processing blocks in this direct form depiction are OQAM pre-processing, synthesis filter bank, the analysis filter bank, and the OQAM post-processing. The transmission channel is classically misplaced when analysing and planning TMUX systems as the channel equalization problem is controlled distinctly. The synthesis and analysis filter banks are naturally the key components. As already mentioned, the Field of filter banks is very broad and even modulated filter banks can be divided into different types depending on the choice of the prototype filters, modulation functions, and desired properties. In this research, we have decided to study M-sub channel filter banks that can be classified. By the following terms:

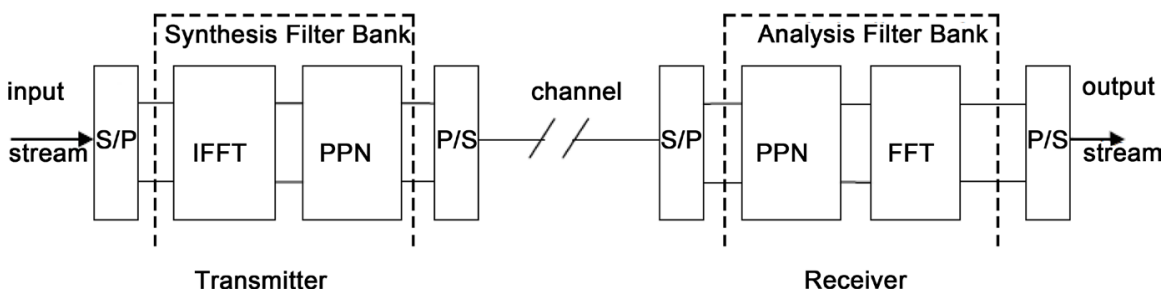

Figure 2. Block diagram of filter bank multi carrier (FBMC) [5] [6]. 


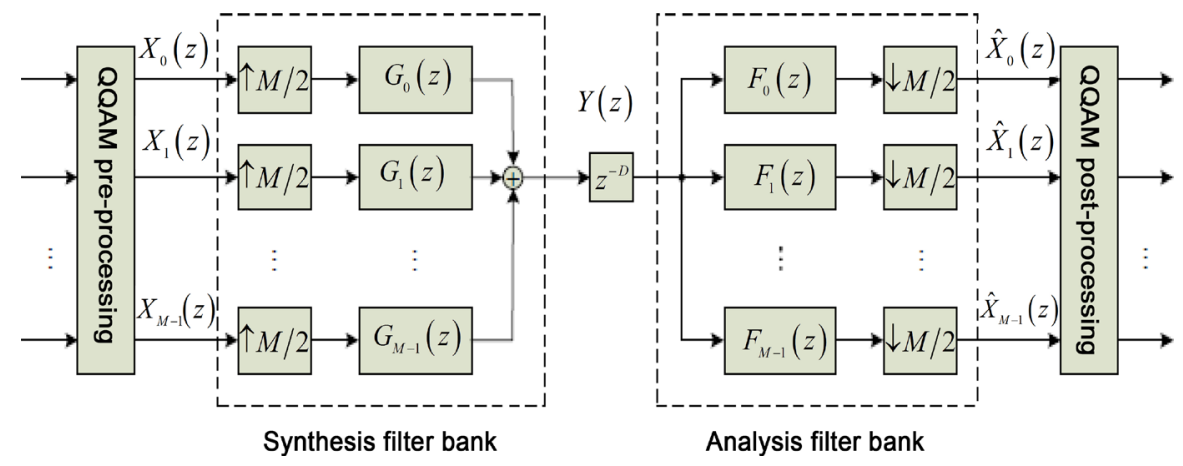

Figure 3. TMUX configuration of filter bank multi-carrier [9].

1) Complex modulated: In order to achieve a good spectral efficiency, a complex I/Q baseband Signal is needed for transmission purposes and complex modulated filter banks are a logical Choice. This means that all sub channel filters are frequency shifted versions of the prototype Filter.

2) Uniform: All sub channel filters have the same bandwidth as the prototype filter and they divide the available channel bandwidth equally.

3) Finite impulse response (FIR): FIR filter banks are used instead of their infinite impulse response (IIR) counterparts because FIR filters are always stable and they are relatively easy to design and implement.

4) Orthogonal: For orthogonal filter banks, only a single prototype filter is needed. Typically, Linear-phase prototype filters are used, and therefore exponential modulation schemes can provide linear-phase sub channel filters. Then the resulting whole system delay is dependable on the order of the prototype filter.

5) Nearly perfect reconstruction (NPR): The output signals are only nearly delayed versions of the input signals, i.e., assured amount of filter bank structure based alterations can be tolerated as long as they are lesser related to those caused by a transmission channel. So the main filter bank design parameters are:

a) The number of sub channels $M$ is essentially a random even number, but characteristically it is a power of two acceptable to deliver effectual application.

b) So the greatest motivating prototype filter lengths are selected to be $L_{p}=K M-1, L_{p}=K M$, and $L_{p}=K M+1$. and, where $K$ is a positive integer called as overlapping factor and it is chosen to be 3 or higher.

c) The prototype filter is intended in such a manner that only instantly adjacent sub channel filters are pointedly overlapping with each other in the frequency domain.

The amount of sub channels is twice the up sampling and down sampling factors suggesting $2 \mathrm{x}$ oversampled filter banks if input and output signals are complex-valued. Hence, if input and output signals are purely real/imaginary-valued then the existing TMUX is comparable to a critically sampled TMUX. This happens because the sample rate (counted in terms of real-valued samples) of the SFB output and AFB input is equal to the sum of the sample rates of the sub channel signals. An extra delay $z^{-D}$, with $D$ depending on the length of the 
prototype filter $\left(L_{p}=K M+1-D\right)$, has to be included either in AFB or SFB output [9].

\subsection{OQAM Pre/Post Processing}

The TMUX system transmits OQAM symbols instead of QAM symbols. The pre-processing block, which utilizes the transformation between QAM and OQAM symbols, is shown in Figure 4. As can be seen, the first operation is a simple complex-to-real conversion, where the real and imaginary parts of a complex-valued symbol $c_{k, l}$ are separated to form two new symbols $d_{k, 2 l}$ and $d_{k, 2 l+1}$ (this operation can also be called as staggering). So the order of these original symbols depends upon the sub channel number, i.e., the conversion is distinct for even and odd numbered sub channels. The complex-to-real conversion upsurges the sample rate by a factor of 2 . After this the second operation is the multiplication by $\theta_{k, n}$ sequence [9].

A possible choice is

$$
\theta_{k, n}=j^{(k+n)}
$$

However, it should be noted that the signs of the $\theta_{k, n}$ sequence can be chosen arbitrarily, but the pattern of real and imaginary samples has to follow the above definition. For example, an alternative sequence

$$
\theta_{k, n}=\left\{\begin{array}{c}
1, j, 1, j \cdots \text { for } k \text { even } \\
j, 1, j, 1 \cdots \text { for } k \text { odd }
\end{array}\right.
$$

The input signals are purely real or imaginary-valued after the OQAM pre-processing.

The post-processing block is shown in Figure 5 and again there are two slightly different structures depending on the sub channel number. The first operation is the multiplication by $\theta_{k, n}^{*}$ sequence that is followed by the operation

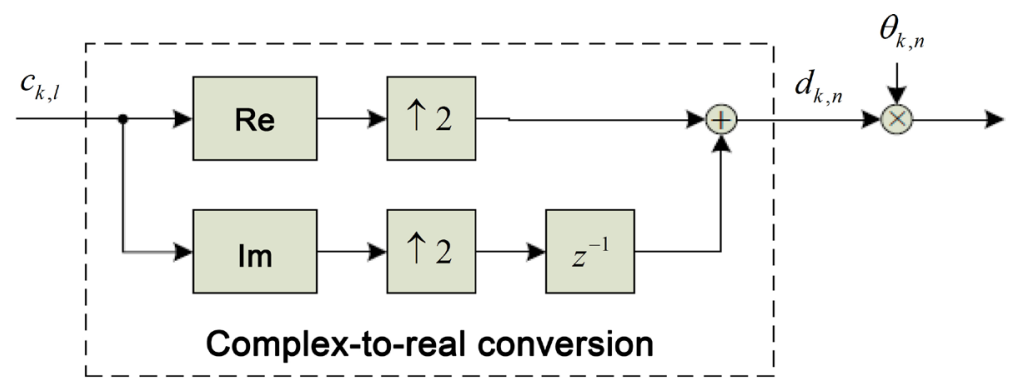

$k$ even

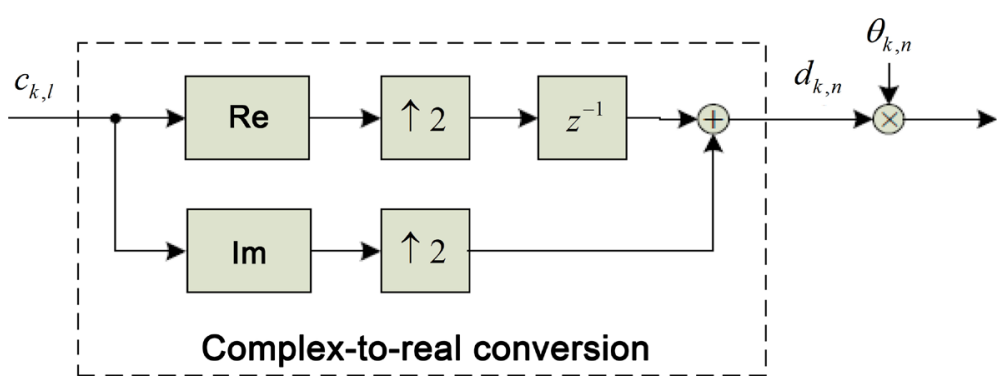

$k$ odd

Figure 4. OQAM preprocessing [9]. 


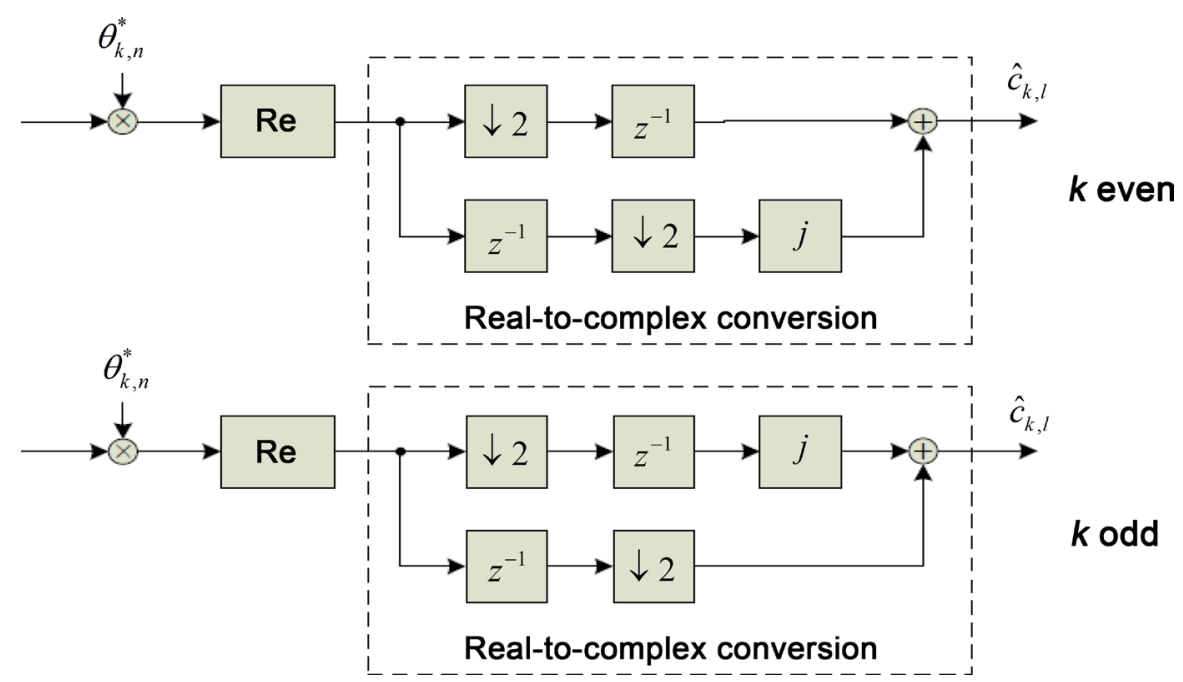

Figure 5. OQAM post processing [9].

of taking the real part. The second operation is real-to complex conversion, in which two successive real-valued symbols (with one multiplied by $j$ ) form a complex-valued symbol $\hat{c}_{k, n}$ (this operation is also called as de-staggering). The real-to-complex conversion decreases the sample rate by a factor 2 . As can be seen, the first operation is a simple complex-to-real conversion, where the real and imaginary parts of a complex-valued symbol $c_{k, l}$ are separated to form two new symbols $d_{k, l}$ and $d_{k, 2 l+1}$ (this operation can also be called as staggering). The order of these new symbols depends on the sub channel number, i.e., the conversion is different for even and odd numbered sub channels. The complex-to-real conversion increases the sample rate by a factor of 2 . The second operation is the multiplication by sequence $\theta_{k, n \text {. }}$ [9].

\subsection{Synthesis and Analysis Filter Banks}

A direct form SFB consists of $M$ up samplers and $M$ synthesis filters. As shown in Figure 3, the input signals $X_{k}(z)$, where $k=0,1,2, \cdots, M-1$, are first up sampled by $\mathrm{M} / 2$ and then filtered with synthesis filters $G_{k}(z)$ The SFB output signal $Y(z)$ is formed when all sub signals are added together. A direct form AFB is constructed using $M$ analysis filters and $M$ down samplers as shown in Figure 3. The input signal $Y(z)$ is first filtered by analysis filters $F_{k}(z)$ and these signals are then down sampled by a factor of $M / 2$ to form output signals $X_{k}(z)$. In the case of chosen class of complex modulated filter banks, all sub channel filters can be generated from a single real-valued linear-phase low pass prototype filter $\mathrm{p}[\mathrm{m}]$ by using exponential modulation. Due to the modulation function, the resulting sub channel filters are complex-valued and therefore the useful frequency range is $[-\pi, \pi]$. Typically, two different types of channel stacking arrangements can be considered, namely, even-stacked filter banks and odd-stacked filter banks. The center frequencies of the $k^{\text {th }}$ sub channel filters are $w_{k}=2 k \pi / M$ and $\omega_{k}=((2 k+1) \pi) / M$ for even stacked and odd-stacked filter banks, respectively. The $k^{\text {th }}$ synthesis filter is given by 


$$
\begin{aligned}
& g_{k}[m]=p[m] \exp \left(j \frac{2 \pi k}{m}\left(m-\frac{L_{p}-1}{2}\right)\right) \\
& m=0,1 \cdots, L_{p}-1
\end{aligned}
$$

where $m=0,1 \cdots, L_{p}-1$ and $L_{p}$ is the filter length. In order to simplify notations, it is assumed that the prototype filter is designed in such a manner that there is no need for the additional scaling factor. The $k^{\text {th }}$ analysis filter is simply a time-reversed and complex-conjugated version of the corresponding synthesis filter

$$
\begin{aligned}
f_{k}[m] & =g_{k}^{*}\left(L_{p}-1-m\right) \\
& =p\left(L_{p}-1-m\right) \exp \left(\frac{2 \pi k}{m}\left(L_{p}\left(1-m-\frac{L_{p}-1}{2}\right)\right)\right) \\
& =p[m] \exp \left(j \frac{2 \pi k}{M}\left(m-\frac{L_{p}-1}{2}\right)\right)
\end{aligned}
$$

An interpretation to the above equations is that first zero-phase sub channel filters are generated from a linear-phase prototype filter and then their impulse responses are delayed by $\left(L_{p}-1\right) / 2$ samples resulting in causal subchannel filters. Due to the nature of the modulation function, the $\mathrm{m}^{\text {th }}$ sub channel filter is purely real and the $M / 2^{\text {th }}$ is purely imaginary. However, all the sub channel filters have linear phase.

\section{Simulation Results}

The analysis compares Filter Bank Multi-Carrier (FBMC) modulation with generic OFDM modulation. FBMC offers ways to overcome the known limitations of OFDM of reduced spectral efficiency and strict synchronization requirements. The benefits have led it to being measured as one of the modulation techniques for $5 \mathrm{G}$ communication structures.

\subsection{Power Spectral Densities of FBMC and OFDM}

Power spectral density of the FBMC transmit signal is designed to give the low out-of-band leakage. In Figure 6 and Figure 7 the plots of the spectral densities for OFDM and FBMC schemes are compared. In Figure 6 it depicts that the FBMC has lesser side lobes. This lets an advanced utilization of the allotted spectrum, leading to advanced spectral efficiency. In Figure 7 the OFDM PSD curve has higher side lobes and out of band leakage. Hence the FBMC scheme is more advantageous in comparison to OFDM by providing higher spectral efficiency. Owing to the per subcarrier filtering, it suffers a larger filter delay (in comparison to UFMC) and also needs OQAM processing.

\subsection{Comparison of Computational Complexity of OFDM and FBMC in Terms of Magnitude Responses}

The computational complexity of filter bank structures can be evaluated by calculating 


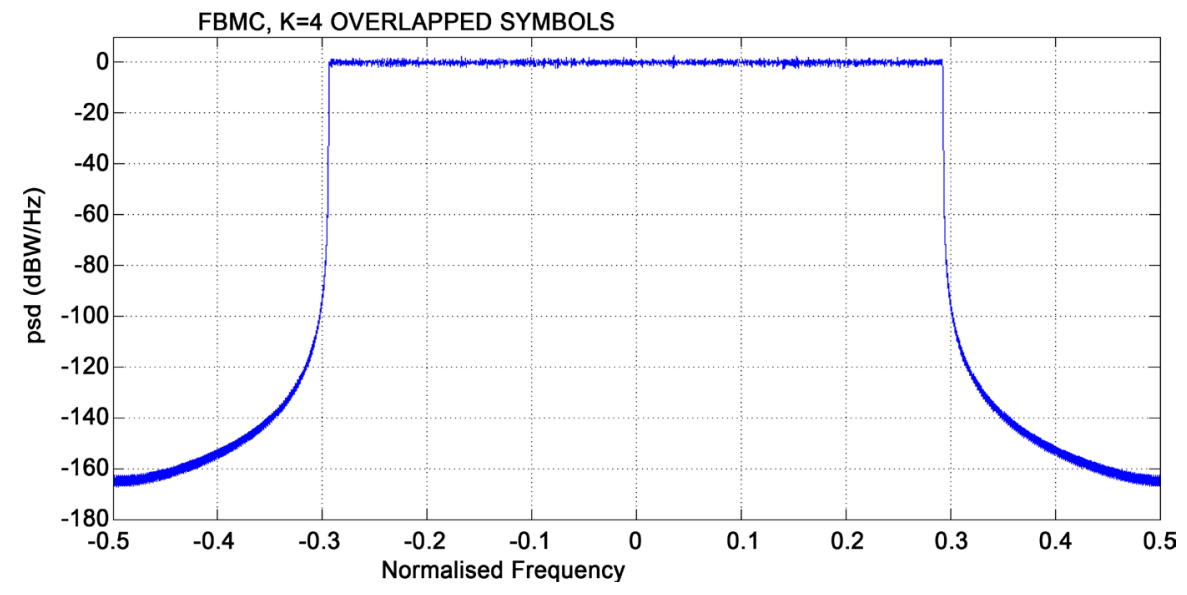

Figure 6. Power spectral density of FBMC.

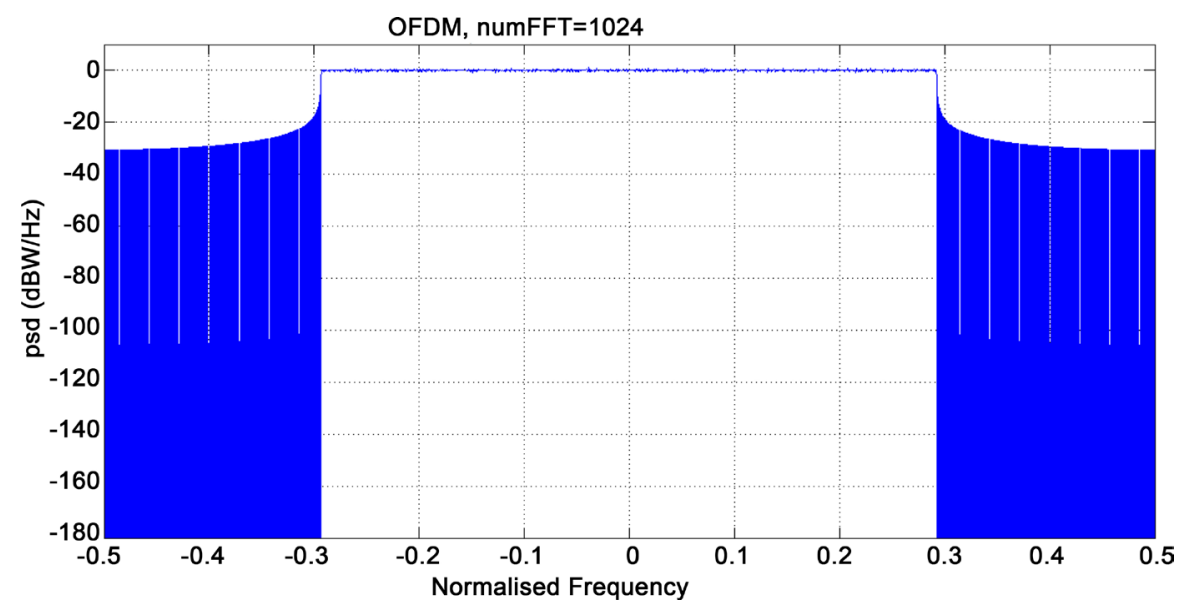

Figure 7. Power spectral density of OFDM.

the number of real multiplications and additions that are necessary to compute a new length-complex-valued output sequence. However, the analysis is just based on the number of multiplications because it is a known fact that adders are considerably cheaper to implement than multipliers. So the Figure 8 depicts that the FBMC is more complex than the conventional OFDM. Here the number of real multiplications as a function of number of sub channels in the case of OFDM and FBMC has been observed. Also when using the polyphase implementations of FBMC the complexity increases as depicted in Figure 8 but decreases when FBMC is implemented without the polyphase.

\subsection{Comparison of Magnitude Responses of Prototype Filters of FBMC and OFDM}

The main difference between FBMC and OFDM is the choice of the prototype filter. Such that OFDM uses a rectangular window filter and FBMC using a prototype filter designed with the Nyquist pulse shaping principle, which can reduce greatly the spectral leakage problem of OFDM. This results in negligible ICI and ISI. In Figure 9 the magnitude responses of prototype filters of FBMC and 


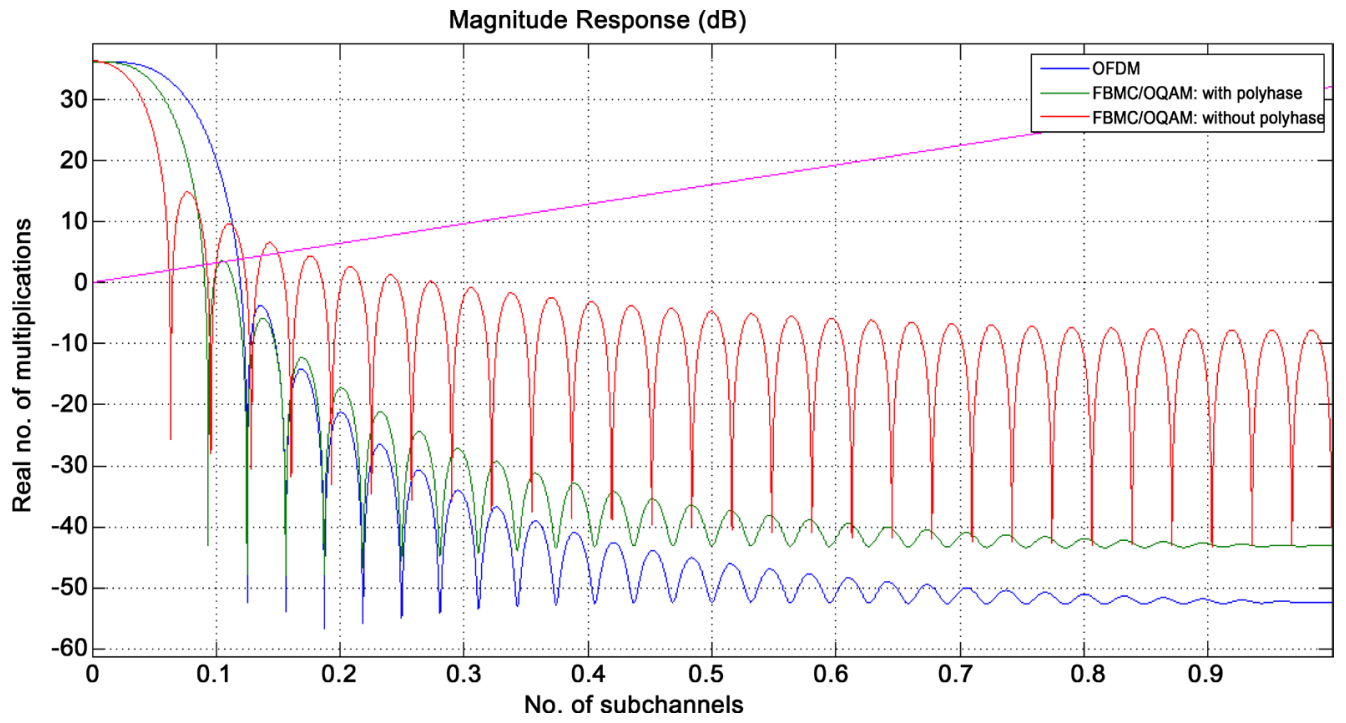

Figure 8. Comparison of computational complexity of FBMC and OFDM in terms of magnitude responses.

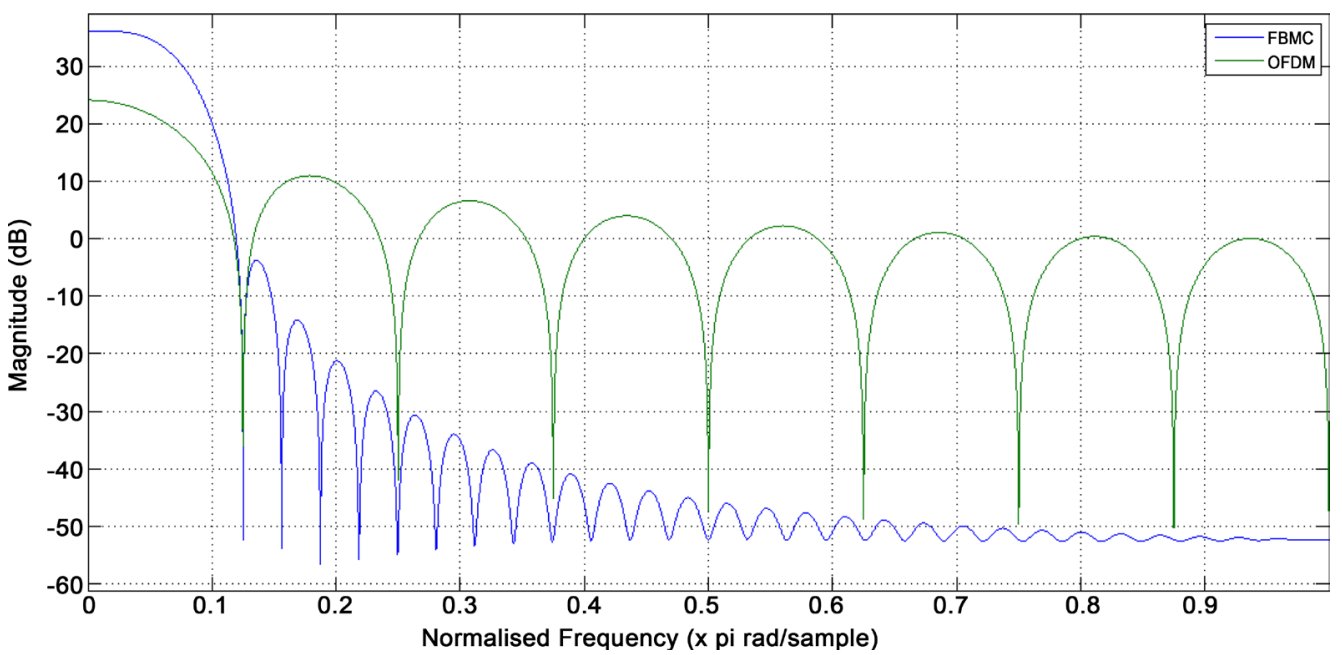

Figure 9. Comparison of magnitude responses of prototype filters of FBMC and OFDM.

OFDM have been compared against the normalized frequency taken on the $\mathrm{x}$ axis.

\section{Conclusion}

In this paper the performance comparison of OFDM and the FBMC as the most potential contenders of $5 \mathrm{G}$ has been carried out and simulated using MATLAB in terms of Power Spectral Density curves of OFDM and FBMC, sub channels of OFDM and FBMC, computational complexities of the two techniques and the prototype filter comparisons of FBMC and OFDM. All the simulations performed show that the FBMC technique is the most promising waveform contender for future wireless communications specially $5 \mathrm{G}$ telecommunications. The shortcomings of OFDM technique have been addressed and removed by the FBMC. FBMC is evolved from OFDM. 


\section{Acknowledgements}

Special thanks to the faculty members of HBTU Kanpur for their guidance and support.

\section{References}

[1] Weiss, T.A. and Jondral, F.K. (2004) Spectrum Pooling: An Innovative Strategy for the Enhancement of Spectrum Efficiency. IEEE Communications Magazine, 42, S8-S14. https://doi.org/10.1109/MCOM.2004.1273768

[2] Schafer, D.J. (2001) Wide Area Adaptive Spectrum Applications. Proceedings of IEEE MILCOM, 1, 1-5. https://doi.org/10.1109/MILCOM.2001.985752

[3] Saltzberg, B. (1967) Performance of an Efficient Parallel Data Transmission System. IEEE Transactions on Communication Technology, 15, 805-811. https://doi.org/10.1109/TCOM.1967.1089674

[4] Farhang Boroujeny, B. (2008) A Square Root Nyquist (M) Filter Design for Digital Communication Systems. IEEE Transactions on Signal Processing, 56, 2127-2132. https://doi.org/10.1109/TSP.2007.912892

[5] Tensubam, B.D., Chanu, N.L. and Singh, S. (2014) Comparative Analysis of FBMC and OFDM Multicarrier Techniques for Wireless Communication Networks. International Journal of Computer Applications, 100, 27-31. https://doi.org/10.5120/17636-8382

[6] Schaich, F. (2010) Filterbank Based Multicarrier Transmission (FBMC)-Evolving OFDM. Bell Labs Alcatel-Lucent.

[7] Hidalgo Stitz, T. (2010) Filterbank Techniques for the Physical Layer in Wireless Communications. Ph.D. Theses, Tampere University of Technology Publications, Tampere, $919 \mathrm{p}$.

[8] Vaidyanathan, P.P. (1993) Multirate Systems and Filterbanks. Prentice Hall, Upper Saddle River.

[9] FP7-ICT Future Networks PHYDYAS-PHYsical Layer for DYnamic AccesS and Cognitive Radio Project (ICT-211887).

http://www.ict-phydyas.org/delivrables/PHYDYAS-D5-1.pdf/view 


\section{Nomenclature}

$\begin{array}{ll}\text { FBMC } & \text { Filter Bank Multi-Carrier } \\ \text { OFDM } & \text { Orthogonal Frequency Division Multiplexing } \\ \text { DSA } & \text { Dynamic Spectrum Access } \\ \text { OQAM } & \text { Offset Quadrature Amplitude Modulation } \\ \text { SFB } & \text { Synthesis Filter Bank } \\ \text { AFB } & \text { Analysis Filter Bank } \\ \text { CP } & \text { Cyclic Prefix in OFDM } \\ \text { MIMO } & \text { Multi Input Multi Output } \\ \text { M } & \text { No. of Subchannels in FBMC Technique } \\ L_{p} & \text { Filter Length in FBMC Technique } \\ K & \text { Overlapping Factor in FBMC Technique } \\ C_{k, l} & \text { Complex Valued Symbol in OQAM Pre/Post Processing } \\ \theta_{k, n} & \text { Sequence Used for Multiplication in OQAM Preprocessing } \\ \theta_{k, n}^{*} & \text { Sequence Used in OQAM Post Processing } \\ X_{k}(z) & \text { Input Signals in FBMC-OQAM System } \\ \ddot{X}_{k}(z) & \text { Output Signals in FBMC-OQAM System } \\ \text { TMUX } & \text { Trans Multiplexer Configuration } \\ G_{k}(z) & \text { Synthesis Filter Used in FBMC-OQAM System } \\ F_{k}(z) & \text { Analysis Filters Used in FBMC-OQAM System } \\ \bar{P}[k] & \text { Prototype Filter Coefficients }\end{array}$

